\title{
Safety aspects of driving freight wagons on 1XTa bogeys with leaf springs when travelling in quasi-static conditions on warped track

\author{
Bezpieczeństwo jazdy wagonów towarowych wyposażonych $w$ wózki \\ 1XTa z resorami piórowymi podczas jazdy w warunkach \\ quasistatycznych przez tory wichrowate
}

\begin{abstract}
This article is a summary of analytical considerations from previous works on the qualification of springs in $1 X T a$ bogies for use in four-axle wagons. The article presents the calculation of an example test drive of wagon in quasi-static conditions through an arc of radius $R=150 \mathrm{~m}$. The results of calculations of the derailment coefficient for rail tankers equipped with four groups of springs of various rigidity were presented along with the conclusions derived from analytical calculations.

Artykut jest podsumowaniem rozważań analitycznych $w$ poprzednich pracach dotyczqcych kwalifikacji resorów w wózkach $1 X$ Ta, przeznaczonych do wagonów czteroosiowych. W artykule przedstawiono obliczenia przejazdu przykładowego wagonu cysterny $w$ warunkach quasi-statycznych przez tuk o promieniu $R=150 \mathrm{~m}$. Przedstawiono wyniki obliczeń wskaźnika wykolejenia dla wagonów cystern wyposażonych $w$ cztery grupy resorów o różnych sztywnościach oraz wnioski z obliczeń analitycznych.
\end{abstract}

\section{INTRODUCTION}

The article is a continuation of analytical work, dedicated to freight wagons on bogey $1 \mathrm{XTa}$ (which does not meet the standard requirements according to the report ORE/ERRIB12/Rp.14/D) [10], equipped with leaf-springs in the primary suspension $[2 \div 4]$. These studies were performed based on the national regulations, namely the $\mathrm{PN}-61 / \mathrm{C}-88171$ standard [13] and the report ORE/ERRI B12 Bericht No.25 [11] as well as the UIC 517, UIC 820 and 821 cards $[6 \div 8]$. From the operational experiences it can be gathered that leaf springs are an important part of the gear system, decisive for drive safety, especially in unfavorable conditions, such as when passing through narrow arcs at their warp limit. The change of the characteristics during operation, resulting from the wear of individual leaf springs, decay of graphite grease on the spring surfaces, corrosion process, etc. is an important phenomenon to consider. It is not uncommon for this process to lead to an increase in the spring rigidity, which results in a reduced wheel load on the warped track. It has also been shown that the leaf spring used in the 1XTa bogey is highly stressed, both in static and fatigue driving conditions. As a result, special attention should be paid to the way springs are assessed. Thus inspection should include WT-2, part 3a-4c, OW-305 [13] and receiver criterion resulting from arrow deflection under load.

\section{WSTĘP}

Artykuł jest kontynuacją prac analitycznych, poświęconych wagonom towarowym na wózkach 1XTa (który nie spełnia wymogów standardowych zgodnie raportem ORE/ERRIB12/Rp.14/D [10], wyposażonych $\mathrm{w}$ resory piórowe $\mathrm{w}$ pierwszym stopniu

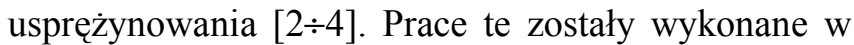
oparciu o przepisy krajowe tzn. norme $\mathrm{PN}-61 / \mathrm{K}$ 88171 [13] oraz tzn. raport ORE/ERRI B12 Bericht

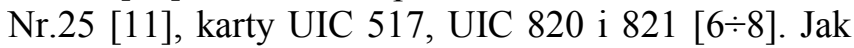
wynika z zebranych doświadczeń eksploatacyjnych resory piórowe są istotnym elementem układu biegowego, decydującym o bezpieczeństwie jazdy, zwłaszcza w niekorzystnych warunkach tzn. podczas przejazdu przez ciasne luki o wichrowatości granicznej.Istotnym zjawiskiem z jakim należy się liczyć, jest zmiana charakterystyki w trakcie eksploatacji, w wyniku zużycia poszczególnych piór resoru, zaniku smaru grafitowego na powierzchniach piór, procesu korozji itd. Wskutek tego procesu, nierzadko dochodzi do zwiększenia sztywności resoru (zmniejszenia podatności), co w efekcie doprowadza do zwiększenia procesu odciążenia koła na torze wichrowatym. Wykazano również, że resor piórowy zastosowany $\mathrm{w}$ wózku 1XTa jest bardzo wytężony, zarówno podczas pracy statycznej jak również pracy zmęczeniowej. W efekcie tego należy szczególną uwagę zwrócić na sposób kwalifikacji resoru. Rewizji należy poddać WT-2, cz.3a-4c, OW-305 [13] oraz kryterium odbiorcze wynikające ze strzałki ugięcia pod obciążeniem. 


\section{SAFETY OF DRIVING RAIL TANKERS THROUGH A WARPED TRACK}

\subsection{General information}

During the passage of rail tankers through a track with maximum permissible warp value, resulting from the national regulations and ORE B55 Rp.8 report [12], the load on the wheels is reduced. Track warp is caused by geometric factors occurring on its trajectory, i.e.:

$>$ the inclination of the ramp outer rail on track curves with warp of up to $2 \%$ and,

$>$ unevenness of the height in the mutual orientation of the rails, the maximum deviation of the highest point on one of the rails from the plane defined by the other three points reaching $15 \mathrm{~mm}$.

Analysis of the derailment safety under quasi-static conditions was carried out on a 406R rail tanker with 1XTa bogies with a base of $2 \mathrm{a}+=2 \mathrm{~m}$ and a base of $2 \mathrm{a}^{*}=6.8 \mathrm{~m}$ with test parameters as defined in the ORE/ERRI B55 Rp.8 report [12].

\subsection{Calculation of the wheel load reduction} $\Delta Q t$ while passing through an arc of radius $R=$ $150 \mathrm{~m}$

Wheel load change $\Delta \mathrm{Qt}$ when passing through a track segment with a maximum warp is expressed by the formula:

$$
\Delta \mathrm{Q}_{\mathrm{t}}=\left(\mathrm{g}^{*}-\mathrm{g}_{\mathrm{G}}\right) \cdot \mathrm{c}_{\mathrm{tA}\left(2 \mathrm{a}^{*}\right)}+\mathrm{g}^{+} \cdot \mathrm{c}_{\mathrm{t}\left(2 \mathrm{a}^{+}\right)}
$$

where:

$\mathrm{g}^{*}$ - warp relative to the wagon base $2 \mathrm{a}^{*}$ (distance between pivots) in \%o,

$\mathrm{g}^{+}-$warp relative to the bogey base $2 \mathrm{a}^{+}$ (distance between wheel sets in bogey) $\mathrm{w}$ $\%$,

$\mathrm{g}_{\mathrm{G}}$ - reduction of vehicle warp $\mathrm{g}^{*}$ due to clearances on side slips in \%o,

$\mathrm{c}_{\mathrm{tA}\left(2 \mathrm{a}^{*}\right)}$ - torsion stiffness of the rail tanker body in $\mathrm{kN} / \%$, $\mathrm{kN} / \%$.

$\mathrm{c}_{\mathrm{A}(2 \mathrm{a}+)}-1 \mathrm{XTa}$ bogey torsion stiffness in

In accordance with ORE/ERRI B55 Rp.8 [12] the warp $\mathrm{g}^{*}$ is expressed as:

$$
\mathrm{g}^{*}=\frac{15}{2 \mathrm{a}^{*}}+2
$$

where:

$$
2 \mathrm{a}^{*} \text { - wagon base size in } \mathrm{m} \text {. }
$$

Reduction of warp due to clearances on the side slips $g_{G}$ can be expressed as:

$$
\mathrm{g}_{\mathrm{G}}=\frac{2 \mathrm{~b}_{\mathrm{A}}}{\mathrm{b}_{\mathrm{G}}} \cdot \frac{\mathrm{d}_{\mathrm{ZGI1}}+\mathrm{d}_{\mathrm{ZGI} 2}+\mathrm{d}_{\mathrm{ZGII1}}+\mathrm{d}_{\mathrm{ZGII} 2}}{2 \cdot 2 \mathrm{a}^{*}}
$$

\section{BEZPIECZEŃSTWO JAZDY WAGONÓW CYSTERN PODCZAS PRZEJAZDU PRZEZ TORY WICHROWATE}

\subsection{Uwagi ogólne}

Podczas przejazdu wagonów cystern przez tory o maksymalnej wichrowatości wynikającej z przepisów krajowych oraz raportu ORE B55 Rp.8 [12] następuje odciążenie kół. Wichrowatość toru jest spowodowana czynnikami geometrycznymi występujacymi na jego trajektorii tzn.:

> pochyleniem rampy przechyłowej toku zewnętrznego na łukach toru $\mathrm{z}$ przechyłką wynoszące maksymalnie $2 \%$ oraz

$>$ nierównościami wysokości we wzajemnym ułożeniu toku szyn maksymalne odchylenie najwyższego punktu znajdującego się na jednej ze szyn od płaszczyzny wyznaczonej przez trzy pozostałe punkty wynoszące $15 \mathrm{~mm}$.

Analize bezpieczeństwa przed wykolejeniem w warunkach quasi-statycznych przeprowadzono na podstawie wagonu cysterny 406R posiadającego wózki 1 XTa o bazie $2 \mathrm{a}^{+}=2 \mathrm{~m}$ i baze $2 \mathrm{a}^{*}=6,8 \mathrm{~m}$ o parametrach badawczych opublikowanych $w$ raporcie ORE/ERRI B55 Rp.8 [12].

\subsection{Obliczenia odciążenia koła $\Delta Q_{t}$ podczas prze- jazdu przez luk o promieniu $R=150 \mathrm{~m}$}

Odciążenie koła $\Delta \mathrm{Q}_{\mathrm{t}}$ podczas przejazdu przez odcinek toru o maksymalnej wichrowatości wyraża się wzorem:

$$
\Delta \mathrm{Q}_{\mathrm{t}}=\left(\mathrm{g}^{*}-\mathrm{g}_{\mathrm{G}}\right) \cdot \mathrm{c}_{{\mathrm{tA}\left(2 \mathrm{a}^{*}\right)}^{*}}+\mathrm{g}^{+} \cdot \mathrm{c}_{\left.\mathrm{t}^{2} \mathrm{a}^{+}\right)}
$$

gdzie:

$\mathrm{g}^{*}$ - wichrowatość pojazdu odniesiona do bazy wagonu $2 \mathrm{a}^{*}$ (odległość pomiędzy czopami skrętu) w \%o,

$\mathrm{g}^{+}$- wichrowatość pojazdu odniesiona do bazy wózka $2 \mathrm{a}^{+}$(odległość pomiędzy zestawami kołowymi w wózku) w \%o,

$\mathrm{g}_{\mathrm{G}}-\mathrm{zmniejszenie}$ wichrowatości pojazdu $\mathrm{g}^{*}$ wskutek zastosowania luzów na ślizgach bocznych $\mathrm{w} \%$,

$\mathrm{c}_{\mathrm{tA}\left(2 \mathrm{a}^{*}\right)}$ - sztywność skrętna nadwozia wagonu cysterny w $\mathrm{kN} / \%$,

$\mathrm{c}_{\mathrm{A}(2 \mathrm{a}+)}$ - sztywność skrętna wózka 1XTa w

$\mathrm{kN} / \%$.

Zgodnie z raportem ORE/ERRI B55 Rp.8 [12] wichrowatość $\mathrm{g}^{*}$ wyraża się wzorem:

$$
\mathrm{g}^{*}=\frac{15}{2 \mathrm{a}^{*}}+2
$$

gdzie: 
where:

$2 b_{A}-$ wheelbase of $1500 \mathrm{~mm}$,

$\mathrm{b}_{\mathrm{G}}$ - distance between side slips and the bogey axis, equal to $850 \mathrm{~mm}$,

$\mathrm{d}_{\mathrm{ZGI} 1}, \mathrm{~d}_{\mathrm{ZGI} 2}, \mathrm{~d}_{\mathrm{ZGII} 1}, \mathrm{~d}_{\mathrm{ZGII} 2}$ - side slip clearances on the first and second bogey.

Warp $\mathrm{g}^{+}$is expressed as:

Warp $\mathrm{g}^{+}$is expressed as:

$$
\mathrm{g}^{+}=7-\frac{5}{2 \mathrm{a}^{+}}
$$

where:

$2 \mathrm{a}^{+}-$bogey base size in $\mathrm{m}$.

After inserting the value $2 a^{*}=6.8 \mathrm{~m}$ to equation (2) it becomes:

$$
\mathrm{g}^{*}=\frac{15}{6.8}+2=4.20 \%
$$

After adding the values for $2 b_{A}=1500 \mathrm{~mm}, b_{G}=850$ $\mathrm{mm}, \mathrm{d}_{\mathrm{ZGII}}, \mathrm{d}_{\mathrm{ZGI} 2}, \mathrm{~d}_{\mathrm{ZGIII}}, \mathrm{d}_{\mathrm{ZGII} 2}=7 \mathrm{~mm}$ into equation (3) and $2 \mathrm{a}^{*}=6800 \mathrm{~mm}$ it becomes:

$$
\mathrm{g}_{\mathrm{G}}=\frac{1500}{850} \cdot \frac{4 \cdot 7}{2 \cdot 6800} \cdot 1000=3.63 \%
$$

By inserting the clearance values $\mathrm{d}_{\mathrm{ZGI}}=\mathrm{d}_{\mathrm{ZGI} 2}=\mathrm{d}_{\mathrm{ZGII} 1}$ $=\mathrm{d}_{\mathrm{ZGII}}=7 \mathrm{~mm}$ the principle that the rigid side slip clearance should be as much as the base of the wagon, but expressed in $\mathrm{mm}$.

After substituting the base of the bogey $2 \mathrm{a}^{+}=2 \mathrm{~m}$ to equation (6) it simplifies to:

$$
\mathrm{g}^{+}=7-\frac{5}{2}=4.5 \%
$$

By inserting the values $\mathrm{c}_{\mathrm{tA}\left(2 \mathrm{a}^{*}\right)}=4.00 \mathrm{kN} / \%$ and $\mathrm{c}_{\mathrm{A}(2 \mathrm{a}+)}=1.08 \mathrm{kN} / \%$ o (based on data collected by PKP and published in ORE/ERRIB55 Rp.8 [12]) to equation (1), along with the remaining calculated values $\mathrm{g}^{*}=4.20 \%, \mathrm{~g}_{\mathrm{G}}=3.63 \%$ and $\mathrm{g}^{+}=4.5 \%$ it becomes:

$$
\Delta \mathrm{Q}_{\mathrm{t}}=(4.20-3.63) \cdot 4+4.5 \cdot 1.08=7.14 \mathrm{kN}
$$

According to the data reported in the ORE/ERRI B55 Rp.8 [12] report, it should be taken into account that the nominal load per wheel is $\overline{\mathrm{Q}}_{0}=292 \mathrm{kN}$.

When analyzing the equation (8), it can be concluded that the total wheel load reduction $\Delta \mathrm{Q}_{\mathrm{t}}$ of the wagon 406R is influenced by:

$>$ track warp on the basis of the wagon equal to $2.28 \mathrm{kN}$, which accounts for $31.9 \%$ of the total wagon load reduction,

$>$ track warp on the basis of the bogey equal to $4.86 \mathrm{kN}$, which accounts for $68.1 \%$ of the total wagon load reduction.

From the presented calculations it can be concluded that the load reduction caused by track warp on the bogey is more significant for this type of a wagon. The wagon-based warp is greatly reduced by the side slip
Zmniejszenie wichrowatości wskutek luzów na ślizgach bocznych $\mathrm{g}_{\mathrm{G}}$ można wyznaczyć ze wzoru:

$$
\mathrm{g}_{\mathrm{G}}=\frac{2 \mathrm{~b}_{\mathrm{A}}}{\mathrm{b}_{\mathrm{G}}} \cdot \frac{\mathrm{d}_{\mathrm{ZGI1}}+\mathrm{d}_{\mathrm{ZGI} 2}+\mathrm{d}_{\mathrm{ZGII}}+\mathrm{d}_{\mathrm{ZGII}}}{2 \cdot 2 \mathrm{a}^{*}}
$$

gdzie:

$2 b_{\mathrm{A}}-$ rozstaw okręgów tocznych wynoszący $1500 \mathrm{~mm}$,

$\mathrm{b}_{\mathrm{G}}$ - odległość ślizgu bocznego od osi wózka, wynoszący $850 \mathrm{~mm}$,

$\mathrm{d}_{\mathrm{ZGI} 1}, \mathrm{~d}_{\mathrm{ZGI} 2}, \mathrm{~d}_{\mathrm{ZGII} 1}, \mathrm{~d}_{\mathrm{ZGII} 2}$ - luzy na ślizgach bocznych na wózku pierwszym oraz drugim.

Wichrowatość $\mathrm{g}^{+}$wyraża się wzorem:

gdzie: $\quad \mathrm{g}^{+}=7-\frac{5}{2 \mathrm{a}^{+}}$

$2 \mathrm{a}^{+}$- baza wózka w $\mathrm{m}$.

Po wstawieniu wartości $2 \mathrm{a}^{*}=6,8 \mathrm{~m}$ do wzoru (2) otrzymuje się:

$$
\mathrm{g}^{*}=\frac{15}{6,8}+2=4,20 \%
$$

Po wstawieniu wartości $2 b_{A}=1500 \mathrm{~mm}, b_{G}=850 \mathrm{~mm}$, $\mathrm{d}_{\mathrm{ZGI} 1}, \mathrm{~d}_{\mathrm{ZGI} 2}, \mathrm{~d}_{\mathrm{ZGIII} 1}, \mathrm{~d}_{\mathrm{ZGII}}=7 \mathrm{~mm}$ do wZoru (3) oraz $2 \mathrm{a}^{*}=6800 \mathrm{~mm}$ otrzymuje się:

$$
\mathrm{g}_{\mathrm{G}}=\frac{1500}{850} \cdot \frac{4 \cdot 7}{2 \cdot 6800} \cdot 1000=3,63 \%
$$

Wstawiając do wzoru (1) wartości $\mathrm{c}_{\mathrm{tA}\left(2 \mathrm{a}^{*}\right.}=4,00 \mathrm{kN} / \%$ o oraz $\mathrm{c}_{\mathrm{A}(2 \mathrm{a}+}=1,08 \mathrm{kN} / \%$ (przyjęta na podstawie danych zebranych przez PKP i opublikowanych w ORE/ERRIB55 Rp.8 [12]) jak również pozostałe wyliczone wartości $\mathrm{g}^{*}=4,20 \%, \mathrm{~g}_{\mathrm{G}}=3,63 \%$ oraz $\mathrm{g}^{+}=4,5 \%$ otrzymuje się:

$$
\Delta \mathrm{Q}_{\mathrm{t}}=(4,20-3,63) \cdot 4+4,5 \cdot 1,08=7,14 \mathrm{kN}
$$

Zgodnie $\mathrm{z}$ danymi podanymi $\mathrm{w}$ raporcie ORE/ERRI B55 Rp.8 [12] należy wziąć pod uwagę, że nacisk nominalny przypadający na jedno koło wynosi $\overline{\mathrm{Q}}_{0}=229,2 \mathrm{kN}$.

Analizując wzór (8) można wyciagnąć wniosek, że na całkowite odciążenie koła $\Delta \mathrm{Q}_{\mathrm{t}}$ wagonu $406 \mathrm{R}$ mają wpływ:

$>$ wichrowatość toru na bazie wagonu wynosząca $2,28 \mathrm{kN}$, co stanowi $31,9 \%$ całkowitego odciążenia wagonu oraz

$>$ wichrowatość toru na bazie wózka wynosząca $4,86 \mathrm{kN}$, co stanowi $68,1 \%$ całkowitego odciążenia wagonu.

Z przedstawionych obliczeń można więc wyciągnąć wniosek, że większe znaczenie dla takiego typu wagonu ma odciążenie spowodowane wichrowatością na bazie wózka. Wichrowatość na bazie wagonu jest znacznie redukowana przez luz na ślizgach bocznych. $\mathrm{W}$ związku $\mathrm{z}$ tym $\mathrm{w}$ dalszej części opracowania przeanalizowano jaki wpływ na odciążenie koła ma zmniejszona podatność resoru (zwiększona sztywność resoru). Wielkość $\mathrm{c}_{\mathrm{tA}(2 \mathrm{a}}{ }^{+}$) określa się wg zależności przedstawionej w raporcie ORE/ERRI B55Rp.8 [12]: 
clearances. Thus, the next part of the article discusses the impact of increased spring rigidity on the wheel load reduction. The $\mathrm{C}_{\mathrm{tA}(2 \mathrm{a}}{ }^{+}$) value is determined according to the relationship shown in the ORE/ERRI B55Rp.8 report [12]:

$$
\frac{1}{\mathrm{c}_{\mathrm{tA}^{2}\left(\mathrm{a}^{+}\right)}}=10^{3}\left[\left(2 \mathrm{~b}_{\mathrm{A}}\right)^{2} \cdot \frac{1}{\mathrm{c}_{\mathrm{t}}^{+}}+\frac{1}{2 \mathrm{a}^{+}} \cdot\left(\frac{\mathrm{b}_{\mathrm{A}}}{\mathrm{b}_{\mathrm{Z}}^{+}}\right)^{2} \cdot \frac{4}{\mathrm{c}_{\mathrm{Z}}^{+}}\right]
$$

where:

$>\mathrm{c}_{\mathrm{t}}^{+}-$torsional rigidity of the bogey frame in $\mathrm{kN} \cdot \mathrm{mm}^{2} / \mathrm{rad} \cdot 10^{10}$,

$>2 \mathrm{~b}_{\mathrm{z}}^{+}-$spring base in the transverse direction in $\mathrm{mm}$ or in $\mathrm{m}$,

$>\mathrm{c}_{\mathrm{Z}}^{+}-$suspension rigidity per wheel (of one spring).

To determine the effect of spring rigidity on the value of $\mathrm{c}_{\mathrm{tA}(2 \mathrm{a})}{ }^{+}$the rigidity of the bogey frame $\mathrm{c}_{\mathrm{t}}^{+}$must be determined using the modified equation (9):

$$
\mathrm{c}_{\mathrm{t}}^{+}=\frac{\left(2 \mathrm{~b}_{\mathrm{A}}\right)^{2}}{\frac{1}{\left.\mathrm{c}_{\mathrm{tA}\left(2 \mathrm{a}^{+}\right)}\right)-\frac{1}{20^{3}}}-\frac{1}{2 \mathrm{a}^{+}}\left(\frac{\mathrm{b}_{\mathrm{A}}}{\mathrm{b}_{\mathrm{Z}}}\right)^{2} \cdot \frac{4}{\mathrm{c}_{\mathrm{Z}}^{+}}}
$$

After inserting the numerical values for $2 b_{A}=1500$ $\mathrm{mm}, \quad \mathrm{c}_{\mathrm{tA}(2 \mathrm{a})}{ }^{+}=1.08 \cdot 10 \quad \mathrm{kN} / \%, \quad 2 \mathrm{a}^{+}=2000 \quad \mathrm{~mm}$, $\mathrm{b}_{\mathrm{z}}=1000 \mathrm{~mm}, \mathrm{c}_{\mathrm{z}}^{+}=1.57 \mathrm{kN} / \mathrm{mm}$ to equation (10) it becomes:

$\mathrm{c}_{\mathrm{t}}^{+}=\frac{(1500)^{2}}{\frac{1}{1.08 \cdot 10^{3}}-\frac{1}{2000}\left(\frac{1500}{2000}\right)^{2} \cdot \frac{4}{1,57}}=\frac{2250000}{2,093654 \cdot 10^{-4}}=1,074676 \cdot 10^{10} \mathrm{kNmm}^{2} / \mathrm{rad}^{-}$

For comparison, the obtained value of $\mathrm{c}_{\mathrm{t}}^{+}$is slightly less than the experimental rigidity of the $\mathrm{Y} 25 \mathrm{Cs}$ bogey frame with a base of $2 \mathrm{a}^{+}$equal to $1.5 \cdot 10^{10} \mathrm{kN} / \mathrm{mm}^{2} \cdot \mathrm{rad}$ [12].

The rigidity $\mathrm{c}_{\mathrm{tA}(2 \mathrm{a})}{ }^{+}$of the $13 \%$ more rigid springs (springs group IV) the relation (9) can be determined by substituting the numerical values for $2 b_{A}=1500$ $\mathrm{mm}, \mathrm{c}_{\mathrm{t}}^{+}=1.074676 \cdot 10^{10} \mathrm{kNmm} / \mathrm{rad}, 2 \mathrm{a}^{+}=2000 \mathrm{~mm}$, $\mathrm{b}_{\mathrm{z}}=1000 \mathrm{~mm}$, and $\mathrm{c}_{\mathrm{z}}^{+}=1.774 \mathrm{kN} / \mathrm{mm}$ as:

$\frac{1}{\mathrm{c}_{\mathrm{tA}\left(2 \mathrm{a}^{+}\right)}}=10^{3}\left[(1500)^{2} \cdot \frac{1}{1,074676 \cdot 10^{10}}+\frac{1}{2000} \cdot\left(\frac{750}{1000}\right)^{2} \cdot \frac{4}{1,774}\right]=0,8434 \% / \mathrm{kN}$

By calculating the reciprocal of the equation (12), the value of $\mathrm{c}_{\mathrm{tA}(2 \mathrm{a})}^{+}$is:

$$
\mathrm{c}_{\mathrm{tA}(2 \mathrm{a})^{+}}=1.1856 \mathrm{kN} / \% \text { o }
$$

The rigidity $\mathrm{C}_{\mathrm{tA}(2 \mathrm{a}}{ }^{+}$for springs with rigidity higher than $17 \%$ (springs group III) the equation (9) can be determined by substituting numerical values for $2 b_{\mathrm{A}}=1500 \mathrm{~mm}, \quad \mathrm{c}_{\mathrm{t}}^{+}=1.074676 \cdot 10^{10} \quad \mathrm{kNmm}^{2} / \mathrm{rad}$, $2 \mathrm{a}^{+}=2000 \quad \mathrm{~mm}, \quad \mathrm{~b}_{\mathrm{z}}=1000 \quad \mathrm{~mm}, \quad$ and $\mathrm{c}_{\mathrm{z}}^{+}=1.8369 \mathrm{kN} / \mathrm{mm}$ into:

$$
\frac{1}{\mathrm{c}_{\mathrm{tA}\left(2 \mathrm{a}^{+}\right)}}=10^{3}\left[\left(2 \mathrm{~b}_{\mathrm{A}}\right)^{2} \cdot \frac{1}{\mathrm{c}_{\mathrm{t}}^{+}}+\frac{1}{2 \mathrm{a}^{+}} \cdot\left(\frac{\mathrm{b}_{\mathrm{A}}}{\mathrm{b}_{\mathrm{Z}}^{+}}\right)^{2} \cdot \frac{4}{\mathrm{c}_{\mathrm{Z}}^{+}}\right]
$$

gdzie:

$>\mathrm{c}_{\mathrm{t}}^{+}-$sztywność skrętna ramy wózka w $\mathrm{kN} \cdot \mathrm{mm}^{2} / \mathrm{rad} \cdot 10^{10}$,

$2 \mathrm{~b}_{\mathrm{z}}^{+}-$baza usprężynowania $\mathrm{w}$ kierunku poprzecznym $\mathrm{w}$ mm lub $\mathrm{w} \mathrm{m}$,

$>\mathrm{c}_{\mathrm{Z}}^{+}-$sztywność usprężynowania przypadająca na jedno koło (jednego resoru).

Aby zbadać wpływ sztywności resoru na wielkość $\mathrm{c}_{\mathrm{tA}(2 \mathrm{a})}{ }^{+}$należy wyznaczyć wartość sztywności ramy wózka $\mathrm{c}_{\mathrm{t}}^{+} \mathrm{z}$ przekształconego wzoru (9):

$$
\mathrm{c}_{\mathrm{t}}^{+}=\frac{\left(2 \mathrm{~b}_{\mathrm{A}}\right)^{2}}{\frac{1}{\mathrm{c}_{\mathrm{tA}\left(2 \mathrm{a}^{+}\right) 10^{3}}}-\frac{1}{2 \mathrm{a}^{+}}\left(\frac{\mathrm{b}_{\mathrm{A}}}{\mathrm{b}_{\mathrm{z}}}\right)^{2} \cdot \frac{4}{\mathrm{c}_{\mathrm{Z}}^{+}}}
$$

Po wstawieniu danych liczbowych $2 b_{A}=1500 \mathrm{~mm}$, $\mathrm{c}_{\mathrm{tA}(2 \mathrm{a})}{ }^{+}=1,08 \cdot 10 \mathrm{kN} / \%, 2 \mathrm{a}^{+}=2000 \mathrm{~mm}, \mathrm{~b}_{\mathrm{z}}=1000 \mathrm{~mm}$, $\mathrm{c}_{\mathrm{z}}^{+}=1,57 \mathrm{kN} / \mathrm{mm}$ do wzoru (10) otrzymuje się:

$\mathrm{c}_{\mathrm{t}}^{+}=\frac{(1500)^{2}}{\frac{1}{1.08 \cdot 10^{3}}-\frac{1}{2000}\left(\frac{1500}{2000}\right)^{2} \cdot \frac{4}{1,57}}=\frac{2250000}{2,093654 \cdot 10^{-4}}=1,074676 \cdot 10^{10} \mathrm{kNmm} / \mathrm{rad}$

Dla porównania otrzymana wartość $\mathrm{c}_{\mathrm{t}}^{+}$jest nieco mniejsza od ustalonej doświadczalnie sztywności skrętnej ramy wózka typu Y25Cs o bazie $2 \mathrm{a}^{+}$ wynoszącej $1,5 \cdot 10^{10} \mathrm{kN} / \mathrm{mm}^{2} \cdot \mathrm{rad}[12]$.

Sztywność $\mathrm{c}_{\mathrm{tA}(2 \mathrm{a})}{ }^{+}$dla resorów sztywniejszych o $13 \%$ (IV grupa resorów) można wyznaczyć z zależności (9) podstawiając dane liczbowe $2 \mathrm{~b}_{\mathrm{A}}=1500 \mathrm{~mm}$, $\mathrm{c}_{\mathrm{t}}^{+}=1,074676 \cdot 10^{10} \quad \mathrm{kNmm} / \mathrm{rad}, \quad 2 \mathrm{a}^{+}=2000 \quad \mathrm{~mm}$, $\mathrm{b}_{\mathrm{z}}=1000 \mathrm{~mm}, \mathrm{c}_{\mathrm{z}}^{+}=1,774 \mathrm{kN} / \mathrm{mm}$ :

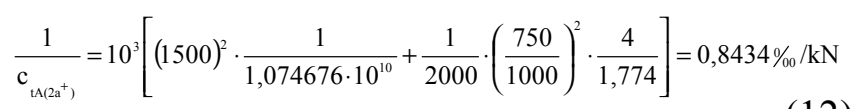

Po wyznaczeniu odwrotności ze wzoru (12), wartość $\mathrm{c}_{\mathrm{tA}(2 \mathrm{a})}^{+}$wynosi:

$$
\mathrm{c}_{\mathrm{tA}(2 \mathrm{a}}{ }^{+}=1,1856 \mathrm{kN} / \%
$$

Sztywność $\mathrm{c}_{\mathrm{tA}(2 \mathrm{a})}{ }^{+}$dla resorów sztywniejszych o $17 \%$ (III grupa resorów) można wyznaczyć z zależności (9) podstawiając dane liczbowe $2 \mathrm{~b}_{\mathrm{A}}=1500 \mathrm{~mm}$, $\mathrm{c}_{\mathrm{t}}^{+}=1,074676 \cdot 10^{10} \quad \mathrm{kNmm} / \mathrm{rad}, \quad 2 \mathrm{a}^{+}=2000 \mathrm{~mm}$, $\mathrm{b}_{\mathrm{z}}=1000 \mathrm{~mm}, \mathrm{c}_{\mathrm{z}}^{+}=1,8369 \mathrm{kN} / \mathrm{mm}$ :

$$
\frac{1}{\mathrm{c}_{\mathrm{tA}\left(2 \mathrm{Z}^{+}\right)}}=10^{3} \cdot\left[(1500)^{2} \cdot \frac{1}{1,074676 \cdot 10^{10}}+\frac{1}{2000} \cdot\left(\frac{750}{1000}\right)^{2} \cdot \frac{4}{1,8369}\right]=0,8218 \% / \mathrm{kN}
$$

Po wyznaczeniu odwrotności ze wzoru (14), wartość $\mathrm{c}_{\mathrm{tA}(2 \mathrm{a})}^{+}$wynosi: 
$\frac{1}{\mathrm{c}_{\mathrm{AA}\left(2 \mathrm{~A}^{+} \mathrm{t}\right.}}=10^{3} \cdot\left[(1500)^{2} \cdot \frac{1}{1,074676 \cdot 10^{10}}+\frac{1}{2000} \cdot\left(\frac{750}{1000}\right)^{2} \cdot \frac{4}{1,8369}\right]=0,8218 \% / \mathrm{kN}$

Once again taking the reciprocal of (14), the value of $\mathrm{c}_{\mathrm{tA}(2 \mathrm{a}}{ }^{+}$can be calculated as:

$$
\mathrm{c}_{\mathrm{tA}(2 \mathrm{a}}^{+}=1.2168 \mathrm{kN} / \% \text { o }
$$

The rigidity $\left.\mathrm{c}_{\mathrm{tA}(2 \mathrm{a}}{ }^{+}\right)$for springs with a $22 \%$ higher rigidity (springs group II) the equation (9) can be determined by substituting numerical values for $2 \mathrm{~b}_{\mathrm{A}}=1500 \mathrm{~mm}, \mathrm{c}_{\mathrm{t}}^{+}=1.074676 \cdot 10^{10} \mathrm{kNmm} / \mathrm{rad}, 2 \mathrm{a}^{+}=$ $2000 \mathrm{~mm}, \mathrm{~b}_{\mathrm{z}}=1000 \mathrm{~mm}$, and $c_{\mathrm{z}}^{+}=1.9154 \mathrm{kN} / \mathrm{mm}$ :

$$
\frac{1}{\mathrm{c}_{\mathrm{AA}\left(2 \mathrm{a}^{+}\right)}}=10^{3}\left[(1500)^{2} \cdot \frac{1}{1,074676 \cdot 10^{10}}+\frac{1}{2000} \cdot\left(\frac{750}{1000}\right)^{2} \cdot \frac{4}{1,9154}\right]=0,7967 \% / \mathrm{kN}
$$

Once again taking the reciprocal of (16), the value of $\mathrm{c}_{\mathrm{tA}(2 \mathrm{a})}^{+}$becomes:

$$
\left.\mathrm{c}_{\mathrm{tA}(2 \mathrm{a}}^{+}\right)=1.2551 \mathrm{kN} / \% \text { o }
$$

The rigidity $\left.\mathrm{c}_{\mathrm{tA}(2 \mathrm{a}}{ }^{+}\right)$for springs with a $26 \%$ higher rigidity (springs group I) the equation (9) can be determined by substituting numerical values for $2 \mathrm{~b}_{\mathrm{A}}=1500 \mathrm{~mm}, \quad \mathrm{c}_{\mathrm{t}}^{+}=1.074676 \cdot 10^{10} \mathrm{kNmm} / \mathrm{rad}$, $2 \mathrm{a}^{+}=2000 \mathrm{~mm}, \mathrm{~b}_{\mathrm{z}}=1000 \mathrm{~mm}$, and $\mathrm{c}_{\mathrm{z}}^{+}=1.9782 \mathrm{~N} / \mathrm{mm}$ :

$$
\frac{1}{\mathrm{c}_{\mathrm{tA}\left(2 \mathrm{a}^{+}\right)}}=10^{3}\left[(1500)^{2} \cdot \frac{1}{1,074676 \cdot 10^{10}}+\frac{1}{2000} \cdot\left(\frac{750}{1000}\right)^{2} \cdot \frac{4}{1,9782}\right]=0,7780 \% / \mathrm{kN}
$$

And the reciprocal of (18), for the value $\left.\mathrm{c}_{\mathrm{tA}(2 \mathrm{a}}{ }^{+}\right)$is:

$$
\mathrm{c}_{\mathrm{tA}(2 \mathrm{a}}{ }^{+}=1.2853 \mathrm{kN} / \% \text { o }
$$

Using formula (1), it is possible to calculate the wheel load change $\Delta Q_{t}$ for all chosen bogey rigidity values $\mathrm{c}_{\mathrm{tA}(2 \mathrm{a}+)}$ applicable for springs with high rigidity. The results have been presented in Tab. 1 .

$$
\left.\mathrm{c}_{\mathrm{tA}(2 \mathrm{a}}{ }^{+}\right)=1,2168 \mathrm{kN} / \%
$$

Sztywność $\mathrm{c}_{\mathrm{tA}(2 \mathrm{a})}{ }^{+}$dla resorów sztywniejszych o $22 \%$ (II grupa resorów) można wyznaczyć z zależności (9) podstawiając dane liczbowe $2 b_{\mathrm{A}}=1500 \mathrm{~mm}$, $\mathrm{c}_{\mathrm{t}}^{+}=1,074676 \cdot 10^{10} \mathrm{kNmm} / \mathrm{rad}, \quad 2 \mathrm{a}^{+}=2000 \mathrm{~mm}$, $\mathrm{b}_{\mathrm{z}}=1000 \mathrm{~mm}, c_{\mathrm{z}}^{+}=1,9154 \mathrm{kN} / \mathrm{mm}$ :

$$
\frac{1}{\mathrm{c}_{\left.{\mathrm{tA}\left(2 \mathrm{C}^{+} \mathrm{t}\right.}\right)}}=10^{3}\left[(1500)^{2} \cdot \frac{1}{1,074676 \cdot 10^{10}}+\frac{1}{2000} \cdot\left(\frac{750}{1000}\right)^{2} \cdot \frac{4}{1,9154}\right]=0,7967 \% 0 \mathrm{kN}
$$

Po wyznaczeniu odwrotności ze wzoru (16), wartość $\left.\mathrm{c}_{\mathrm{tA}(2 \mathrm{a}}{ }^{+}\right)$Wynosi:

$$
\mathrm{c}_{\mathrm{tA}(2 \mathrm{a}}{ }^{+}=1,2551 \mathrm{kN} / \%
$$

Sztywność $\mathrm{c}_{\mathrm{tA}(2 \mathrm{a})}{ }^{+}$dla resorów sztywniejszych o $26 \%$ (I grupa resorów) można wyznaczyć z zależności (9) podstawiając dane liczbowe $2 b_{\mathrm{A}}=1500 \mathrm{~mm}$, $\mathrm{c}_{\mathrm{t}}^{+}=1,074676 \cdot 10^{10} \mathrm{kNmm} / \mathrm{rad}, \quad 2 \mathrm{a}^{+}=2000 \mathrm{~mm}$, $\mathrm{b}_{\mathrm{z}}=1000 \mathrm{~mm}, \mathrm{c}_{\mathrm{z}}^{+}=1,9782 \mathrm{~N} / \mathrm{mm}$ :

$$
\frac{1}{\left.\mathrm{c}_{\mathrm{t}\left(\mathrm{R}\left(\mathrm{A}^{+}\right)\right.}\right)}=10^{3}\left[(1500)^{2} \cdot \frac{1}{1,074676 \cdot 10^{10}}+\frac{1}{2000} \cdot\left(\frac{750}{1000}\right)^{2} \cdot \frac{4}{1,9782}\right]=0,7780 \% / \mathrm{kN}
$$

Po wyznaczeniu odwrotności ze wzoru (18), wartość $\left.\mathrm{c}_{\mathrm{tA}(2 \mathrm{a}}{ }^{+}\right)$wynosi:

$$
\left.\mathrm{c}_{\mathrm{tA}(2 \mathrm{a}}{ }^{+}\right)=1,2853 \mathrm{kN} / \% \text { o }
$$

Korzystając ze wzoru (1) można wyznaczyć odcią-

\begin{tabular}{|c|c|c|c|c|c|c|c|c|c|}
\hline $\begin{array}{c}\text { Sztywność } \\
\text { resoru } \\
{[\mathrm{kN} / \mathbf{m m}]}\end{array}$ & $\begin{array}{c}\mathrm{g}^{*} \\
{[\%]}\end{array}$ & $\begin{array}{c}\mathbf{d}_{\mathrm{zg}} \\
{[\mathrm{mm}]}\end{array}$ & $\underset{[\%]}{\mathbf{g}_{\mathrm{G}}}$ & $\begin{array}{l}\mathbf{g}^{+} \\
{[\% \circ]}\end{array}$ & $\begin{array}{c}c_{\mathrm{tA}\left(2 \mathrm{a}^{*}\right)} \\
{[\mathrm{kN} / \% \mathrm{\%}]}\end{array}$ & ${ }_{[\mathrm{kN} / \% \mathrm{oo}]}^{\left.\mathrm{c}_{\mathrm{t}(2 \mathrm{a}}{ }^{+}\right)}$ & $\begin{array}{l}\Delta \mathbf{Q}_{\mathrm{t}} \\
{[\mathrm{kN}]}\end{array}$ & $\begin{array}{c}\Delta Q_{\mathrm{t}} \\
\text { udzial } \\
\text { wózka } \\
{[\mathrm{kN}]}\end{array}$ & $\begin{array}{r}\Delta \mathbf{Q}_{\mathrm{t}} \\
\text { udzia } \\
\text { pudla } \\
{[\mathrm{kN}]}\end{array}$ \\
\hline \multirow{3}{*}{$\begin{array}{c}c=0,636 \\
\text { (nominalna) }\end{array}$} & 4,20 & 7 & 3,63 & 4,5 & 4,00 & 1,08 & 7,14 & 4,86 & 2,28 \\
\hline & 4,20 & 8 & 4,15 & 4,5 & 4,00 & 1,08 & 5,06 & 4,86 & 0,2 \\
\hline & 4,20 & 9 & 4,66 & 4,5 & 4,00 & 1,08 & 4,86 & 4,86 & - \\
\hline \multirow{3}{*}{$\begin{array}{c}\mathrm{c}=0,563 \\
\text { (IV grupa } \\
\text { resorów) } \\
\end{array}$} & 4,20 & 7 & 3,63 & 4,5 & 4,00 & 1,18 & 7,59 & 5,31 & 2,28 \\
\hline & 4,20 & 8 & 4,15 & 4,5 & 4,00 & 1,18 & 5,51 & 5,31 & 0,2 \\
\hline & 4,20 & 9 & 4,66 & 4,5 & 4,00 & 1,18 & 5,31 & 5,31 & - \\
\hline \multirow{3}{*}{$\begin{array}{c}\mathrm{c}=0,544 \\
\text { (III grupa } \\
\text { resorów) }\end{array}$} & 4,20 & 7 & 3,63 & 4,5 & 4,00 & 1,21 & 7,72 & 5,44 & 2,28 \\
\hline & 4,20 & 8 & 4,15 & 4,5 & 4,00 & 1,21 & 5,64 & 5,44 & 0,2 \\
\hline & 4,20 & 9 & 4,66 & 4,5 & 4,00 & 1,21 & 5,44 & 5,44 & - \\
\hline \multirow{3}{*}{$\begin{array}{c}\mathrm{c}=0,522 \\
\text { (II grupa } \\
\text { resorów) }\end{array}$} & 4,20 & 7 & 3,63 & 4,5 & 4,00 & 1,25 & 7,90 & 5,62 & 2,28 \\
\hline & 4,20 & 8 & 4,15 & 4,5 & 4,00 & 1,25 & 5,82 & 5,62 & 0,2 \\
\hline & 4,20 & 9 & 4,66 & 4,5 & 4,00 & 1,25 & 5,62 & 5,62 & - \\
\hline \multirow{3}{*}{$\begin{array}{l}\mathrm{c}=0,5055 \\
\text { (I grupa } \\
\text { resorów) }\end{array}$} & 4,20 & 7 & 3,63 & 4,5 & 4,00 & 1,28 & 8,04 & 5,76 & 2,28 \\
\hline & 4,20 & 8 & 4,15 & 4,5 & 4,00 & 1,28 & 5,96 & 5,76 & 0,2 \\
\hline & 4,20 & 9 & 4,66 & 4,5 & 4,00 & 1,28 & 5,76 & 5,76 & - \\
\hline
\end{tabular}
żenia kół $\Delta \mathrm{Q}_{\mathrm{t}}$ dla wszystkich wybranych sztywności wózka $c_{\mathrm{tA}(2 \mathrm{a}+)}$ obowiązujących dla resorów o większej sztywności. Wyniki obliczeń przedstawiono w tablicy 1.

Comparison of wheel load change $\Delta Q_{t}$ calculation results for a „406R” type wagon with a bogey equipped with varied rigidity springs and in relation to side slip clearances

Zestawienie wyników obliczeń odciążenia koła $\Delta Q_{t}$ dla wagonu „406R” z wózkami z resorami o większej sztywności i w zależności od luzów na ślizgach bocznych

Tablica 1 
From the results of the calculations presented in Table 1 , it can be concluded that reduced rigidity of springs (increased stiffness), which contributes to the reduction of load on the wagon wheels on the warped track, can be compensated for by the increase in clearance on the side slips. This increase reduces the proportion of wagon body torsional rigidity and compensates for the increased proportion of warp in the wheel load reduction. The construction procedure would be to adjust the clearances on side slips in the upper tolerance limit, i.e. $7^{+2} \mathrm{~mm}=9 \mathrm{~mm}$ for a $6.8 \mathrm{~m}$ wagon. Using the assumption that the base load (with a uniform distribution of all wheel loads in the 406R rail tanker on a $1 \mathrm{XTa}$ bogey) this criterion of derailment is the so-called Nadal criterion, expressed by the formula:

$$
\left(\frac{\mathrm{Y}}{\mathrm{Q}_{\mathrm{IRZECZ}}}\right)_{Y=70^{0}} \leq 1.2
$$

This criterion applies to wheels with a skew angle of the rim equal to $\gamma=70$ ? and a friction coefficient between the wheel and the rail of $\mu=0.36$ (on dry rails).

The value of 1.2 is the result of the following equation after substituting the values for $\gamma=70^{\circ}$ and $\mu=0.36$ :

$$
\frac{\mathrm{Y}}{\mathrm{Q}_{\text {IRZECZ }}}=\frac{\operatorname{tg} \gamma-\mu}{1+\mu \cdot \operatorname{tg} \gamma}=\frac{\operatorname{tg} 70^{\circ}-0.36}{1+0.36 \cdot \operatorname{tg} 70^{\circ}}=1.2
$$

\subsection{Calculation of transverse forces $Y_{a}$ and $Y_{i}$} acting on the outer and inner path of the track

The transverse forces acting on the wheel moving on the outer rail $Y_{a}$ and on the wheel moving on the inner rail $Y_{i}$ can be calculated using formulas as described in ORE/ERRI B55 Rp.8 [12]:

The coefficients for equation (22) for the transverse forces $Y_{a}$ and $Y_{i}$ are given in table 2 .
$\mathrm{Z}$ wyników obliczeń przedstawionych $\mathrm{w}$ tablicy 1 można wyciągnąć wniosek, że zmniejszoną podatność resorów (zwiększoną sztywność), która wpływa na wzrost odciążenia koła wagonu cysterny na wichrowatym torze można rekompensować wzrostem luzów na ślizgach bocznych. Wzrost ten powoduje zmniejszenie udziału sztywności skrętnej nadwozia i rekompensuje zwiększony udział wichrowatości wózka w odciążeniu zestawu kołowego. Zabieg konstrukcyjny polegałby na regulacji luzów na ślizgach bocznych $\mathrm{w}$ górnym polu tolerancji tzn. $7^{+2} \mathrm{~mm}=9 \mathrm{~mm}$ dla wagonu $6,8 \mathrm{~m}$. Wychodząc $\mathrm{z}$ założenia, że nacisk bazowy (przy równomiernym rozkładzie nacisków wszystkich kół w wagonie cysternie 406R na wózkach 1XTa) to kryterium wykolejenia czyli tzw. kryterium Nadala wyraża się wzorem:

$$
\left(\frac{\mathrm{Y}}{\mathrm{Q}_{\mathrm{RZECCZ}}}\right)_{\gamma=70^{0}} \leq 1,2
$$

Kryterium to obowiązuje dla kół z kątem pochylenia obrzeża $\gamma=70$ ? oraz współczynnika tarcia między kołem a szyną wynoszącym $\mu=0,36$ (na suchych szynach).

Wartość 1,2 wynika $\mathrm{z}$ poniższej zależności po podstawieniu $\gamma=70^{\circ}$ oraz $\mu=0,36$ :

$$
\frac{\mathrm{Y}}{\mathrm{Q}_{\text {IRZECZ }}}=\frac{\operatorname{tg} \gamma-\mu}{1+\mu \cdot \operatorname{tg} \gamma}=\frac{\operatorname{tg} 70^{\circ}-0,36}{1+0,36 \cdot \operatorname{tg} 70^{\circ}}=1,2
$$

\subsection{Obliczenie sil poprzecznych $Y_{a}$ i $Y_{i}$ dzialających na zewnętrzny i wewnętrzny tok toru}

Siły poprzeczne działające na koło, znajdujące się na zewnętrznym toku szynowym $Y_{a}$ oraz na koło, znajdujące się na wewnętrznym toku szynowym $Y_{i}$ można wyznaczyć ze wzorów na podstawie raportu ORE/ERRI B55 Rp.8 [12]:

$$
\mathrm{Y}=\left\{\mathrm{Qx}\left[\mathrm{m}_{1} \cdot \mathrm{a}_{\mathrm{q}}+\mathrm{m}_{2}+\mathrm{m}_{3} \cdot \mathrm{a}_{\mathrm{q}}+\mathrm{m}_{4}\right]+\mathrm{x} \cdot\left(\mathrm{c}_{1} \cdot \mathrm{a}_{\mathrm{q}}+\mathrm{c}_{2}\right)+\mathrm{c}_{3} \cdot \mathrm{a}_{\mathrm{q}}+\mathrm{c}_{4}\right\} \cdot 10^{-3}
$$

The coefficients for determining the transverse forces $Y_{a}$ and $Y_{i}$ as described in ORE/ERRI B12 Rp.8 [12] Wspólczynniki do wyznaczania sil poprzecznych $Y_{\mathrm{a}}$ oraz $Y_{\mathrm{i}}$ wg raportu ORE/ERRI B12 Rp.8 [12]

Tablica 2

\begin{tabular}{|c|c|c|c|c|c|c|c|c|c|}
\hline L.p. & $\mathrm{Y}$ & $\mathrm{m}_{1}$ & $\mathrm{~m}_{2}$ & $\mathrm{~m}_{3}$ & $\mathrm{~m}_{4}$ & $\mathrm{c}_{1}$ & $\mathrm{c}_{2}$ & $\mathrm{c}_{3}$ & $\mathrm{c}_{4}$ \\
\hline $\mathbf{1 .}$ & $\mathrm{Y}_{\mathrm{a}}$ & $\mathbf{5 , 7 1 6}$ & $\mathbf{- 2 5 , 7}$ & $\mathbf{- 8 9 , 6 2 3}$ & $\mathbf{8 4 6 , 6 2 5}$ & $\mathbf{- 1 7 0 , 5 9 3}$ & $\mathbf{7 , 8 3 7}$ & $\mathbf{3 6 0 9 , 7 5 3}$ & $\mathbf{1 8 1 0 , 2 7 7}$ \\
\hline $\mathbf{2 .}$ & $\mathbf{Y}_{\mathbf{i}}$ & $\mathbf{- 5 , 5 0 6}$ & $\mathbf{2 0 , 5 8 6}$ & $\mathbf{2 7 0 , 4 8 9}$ & $\mathbf{- 7 4 4 , 3 6 6}$ & $\mathbf{2 5 2 , 7 6 5}$ & $\mathbf{- 1 5 2 , 7 8 4}$ & $\mathbf{- 6 1 2 5 , 2 5 9}$ & $\mathbf{1 7 9 3 , 5 3 7}$ \\
\hline
\end{tabular}

The formula and coefficients are based on tests for freight wagons with standard bogeys of the Y25 family carried out at the German Railway Research and Test Center in Minden.

Substituting numerical values, i.e. $\mathrm{x}=150^{0,5}=12.24$ and $\overline{\mathrm{Q}}_{0}^{\mathrm{j}}=29.2 \mathrm{kN}, \mathrm{a}_{\mathrm{q}}=0 \mathrm{~m} / \mathrm{s}^{2}$ into the equations results in:
Współczynniki do równania (22) dla sił poprzecznych $Y_{a}$ oraz $Y_{i}$ są podane $w$ tablicy 2.

Wzór oraz współczynniki powstał w oparciu o badania dla wagonów towarowych $\mathrm{z}$ wózkami standardowymi rodziny Y25 przeprowadzone w Ośrodku Prób i Badań kolei niemieckich DB w Minden.

Po wstawieniu danych liczbowych tzn. $x=150^{0,5}=12,24$ oraz $\mathrm{Q}_{0}^{-}=29,2 \mathrm{kN}, \mathrm{a}_{\mathrm{q}}=0 \mathrm{~m} / \mathrm{s}^{2}$ otrzymuje się: 


$$
\begin{aligned}
& Y_{a}=\left\{\begin{array}{l}
29,2 \cdot[12,24 \cdot(5,716 \cdot 0-25,7)-89,623 \cdot 0+846,625]+12,24 \cdot(-170,593 \cdot 0+7,837)+ \\
3609,753 \cdot 0+1810,277
\end{array}\right\} \cdot 10^{-3} \\
& Y_{a}=17,442 \mathrm{kN} \\
& Y_{i}=\left\{\begin{array}{l}
29,2 \cdot[12,24 \cdot(-5,506 \cdot 0+20,586)+270,489 \cdot 0-744,366]+12,24 \cdot(252,765 \cdot 0-152,784)- \\
6125,259 \cdot 0+1793,537
\end{array}\right\} \cdot 10^{-3} \\
& Y_{i}=-14,454 \mathrm{kN}
\end{aligned}
$$

\subsection{Calculation of the total wheel load reduction} while passing through warped track

Actual wheel load reduction (reduction of vertical pressure of the wheel on the rail) is:

$$
\Delta \mathrm{Q}_{\text {trzecz }}=\Delta \mathrm{Q}_{\mathrm{t}}+\Delta \mathrm{Q}_{\mathrm{fzo}} \pm \Delta \mathrm{Q}_{\mathrm{fy}}
$$

As stated in ORE/ERRI B55 Rp.8[12]:

$$
\Delta \mathrm{Q}_{\mathrm{fzo}}=\Delta \mathrm{Q}_{\mathrm{fu}}+\Delta \mathrm{Q}_{\mathrm{t}}+\Delta \mathrm{Q}_{\text {eаo }}+\Delta \mathrm{Q}_{\text {ebo }}
$$

where:

$\Delta \mathrm{Q}_{\mu}$ - wheel load reduction due to the friction effects in suspension friction dampers (first degree),

$\Delta \mathrm{Q}_{\mathrm{t}}$ - wheel load reduction due to the warp of the wagon itself,

$\Delta \mathrm{Q}_{\text {eao }}$ - wheel load reduction due to the eccentricity of the wagon center of mass in the longitudal direction,

$\Delta \mathrm{Q}_{\text {ebo }}$ - wheel load reduction due to the eccentricity of the wagon center of mass in the transverse direction.

After adding the relation:

$$
\Delta \mathrm{Q}_{\mathrm{fzo}}=\mathrm{q}_{0} \cdot \overline{\mathrm{Q}}_{0}
$$

where:

$\mathrm{q}_{0}$ - the relative coefficient of wheel load reduction for empty freight wagons

After adding the relation:

$$
\Delta \mathrm{Q}_{\mathrm{fy}}= \pm \frac{\mathrm{Y}_{\mathrm{a}}-\mathrm{Y}_{\mathrm{i}}}{2 \mathrm{~b}_{\mathrm{A}}} \cdot \mathrm{h}_{F}
$$

where:

$$
h_{F}-\text { wheel radius. }
$$

After inserting numerical values $\overline{\mathrm{Q}}_{0}=29.2 \mathrm{kN}$, $\Delta \mathrm{Q}_{\mathrm{t}}=7.14 \mathrm{kN}, \mathrm{Y}_{\mathrm{a}}=17.442 \mathrm{kN}, \mathrm{Y}_{\mathrm{i}}=-14.454 \mathrm{kN}, \mathrm{h}_{\mathrm{F}}=0.460$ $\mathrm{m}$ and $2 \mathrm{~b}_{\mathrm{A}}=1.5 \mathrm{~m}$ to equation (26) becomes:

$$
\Delta \mathrm{Q}_{\mathrm{tz}}=7.14+0.2 \cdot 29.2+\frac{17.442-14.454}{1.5} \cdot 0.460=13.89 \mathrm{kN}
$$

Actual vertical load on the rail in this case is:

$$
\mathrm{Q}_{\mathrm{Iz}}=\overline{\mathrm{Q}}_{0}-\Delta \mathrm{Q}_{\mathrm{trz}}=29,2-13,89=15,31 \mathrm{kN}
$$

\subsection{Derailment coefficient calculation for a wagon with springs of different rigidity}

The derailment coefficient in this case, when substituting the numerical data i.e. $\mathrm{Q}_{\mathrm{rz}}=15.31 \mathrm{kN}$ and $\mathrm{Y}_{\mathrm{a}}=17.442 \mathrm{kN}$ equals:

\subsection{Obliczenie całkowitego odciążenia koła podczas} przejazdu przez tory wichrowate

Rzeczywiste odciążenie koła (zmniejszenie nacisku pionowego koła na szynę) wynosi:

$$
\Delta \mathrm{Q}_{\text {trrecz }}=\Delta \mathrm{Q}_{\mathrm{t}}+\Delta \mathrm{Q}_{\mathrm{fzo}} \pm \Delta \mathrm{Q}_{\mathrm{fy}}
$$

Zgodnie $\mathrm{z}$ raportem ORE/ERRI B55 Rp.8[12]:

$$
\Delta \mathrm{Q}_{\mathrm{fzo}}=\Delta \mathrm{Q}_{\mathrm{fu}}+\Delta \mathrm{Q}_{\mathrm{t}}+\Delta \mathrm{Q}_{\text {cao }}+\Delta \mathrm{Q}_{\text {ebo }}
$$

gdzie:

$\Delta \mathrm{Q}_{\mu}$ - odciążenie koła $\mathrm{z}$ tytułu tarcia $\mathrm{w}$ tłumikach ciernych usprężynowania przymaźnicznego (pierwszego stopnia),

$\Delta \mathrm{Q}_{\mathrm{t}}-$ odciążenie koła $\mathrm{z}$ tytułu zwichrowania własnego wagonu,

$\Delta \mathrm{Q}_{\text {eao }}$ - odciążenie koła $\mathrm{z}$ tytułu ekscentryczności środka ciężkości (masy) wagonu w kierunku wzdłużnym,

$\Delta Q_{\text {ebo }}$ - odciążenie koła z tytułu ekscentryczności środka ciężkości wagonu (masy) w kierunku poprzecznym.

Po wstawieniu zależności:

$$
\Delta \mathrm{Q}_{\mathrm{fzo}}=\mathrm{q}_{0} \cdot \overline{\mathrm{Q}}_{0}
$$

gdzie:

$\mathrm{q}_{0}$ - relatywny współczynnik odciążenia koła dla wagonów towarowych w stanie próżny

Po wstawieniu zależności:

$$
\Delta \mathrm{Q}_{\mathrm{fy}}= \pm \frac{\mathrm{Ya}_{\mathrm{a}}-\mathrm{Y}_{\mathrm{i}}}{2 \mathrm{~b}_{\mathrm{A}}} \cdot \mathrm{h}_{F}
$$

gdzie:

$\mathrm{h}_{\mathrm{F}}-$ promień toczny koła.

Po wstawieniu danych liczbowych $\overline{\mathrm{Q}}_{0}=29,2 \mathrm{kN}$, $\Delta \mathrm{Q}_{\mathrm{t}}=7,14 \mathrm{kN}, \mathrm{Y}_{\mathrm{a}}=17,442 \mathrm{kN}, \mathrm{Y}_{\mathrm{i}}=-14,454 \mathrm{kN}, \mathrm{h}_{\mathrm{F}}=0,460$ $\mathrm{m}$ oraz $2 \mathrm{~b}_{\mathrm{A}}=1,5 \mathrm{~m}$ do wzoru (26) otrzymuje się:

$$
\Delta \mathrm{Q}_{\mathrm{tzz}}=7,14+0,2 \cdot 29,2+\frac{17,442-14,454}{1,5} \cdot 0,460=13,89 \mathrm{kN}
$$

Rzeczywisty nacisk pionowy koła na szynę w takim przypadku wvnosi:

$$
\mathrm{Q}_{\mathrm{Tz}}=\overline{\mathrm{Q}}_{0}-\Delta \mathrm{Q}_{\mathrm{trz}}=29,2-13,89=15,31 \mathrm{kN}
$$

2.5. Obliczenia wskaźnika wykolejenia dla przejazdu wagonu $\mathrm{z}$ resorami o różnych sztywnościach

Wskaźnik wykolejenia w takim przypadku, przy podstawieniu danych liczbowych tzn. $\mathrm{Q}_{\mathrm{rz}}=15,31 \mathrm{kN}$ oraz $\mathrm{Y}_{\mathrm{a}}=17,442 \mathrm{kN}$ wynosi: 


$$
\frac{Y_{a}}{Q_{r z}}=\frac{Y_{a}}{Q_{o}-\Delta Q_{t}}=\frac{17.442}{29.2-13.89}=1.13
$$

The derailment coefficient is lower than for a drive in quasi-static conditions i.e. 1.2.

The relative load reduction $\Delta \mathrm{q}$ is:

$$
\Delta \mathrm{q}=\frac{\Delta \mathrm{Q}_{\mathrm{r}}}{\overline{\mathrm{Q}}_{0}}=\frac{29.2-15.31}{29.2}=0.47
$$

The boundary of the relative wheel load reduction lim $\Delta q$ equals:

$$
\lim \Delta \mathrm{q}=\frac{\lim \left(\frac{\mathrm{Y}}{\mathrm{Q}}\right)_{\mathrm{a}}-\frac{\mathrm{Y}_{\mathrm{a}}}{\overline{\mathrm{Q}}_{0}}}{\lim \left(\frac{\mathrm{Y}}{\mathrm{Q}}\right)_{\mathrm{a}}}=\frac{1.2-\frac{17.442}{29.2}}{1.2}=\frac{1.2-0.59}{1.2}=0.50
$$

The condition $\Delta \mathrm{q}<\lim \Delta \mathrm{q}$ is met.

By completing the equation (20) with numbers

$\overline{\mathrm{Q}}_{0}=29,2 \mathrm{kN}, \Delta \mathrm{Q}_{\mathrm{trzecz}}=13,89 \mathrm{kN}$ (nominal spring rigidity), $\mathrm{Y}_{\mathrm{a}}=17.442 \mathrm{kN}$ results in:

$$
\left(\frac{17.442}{29.2-13.89}\right)=1.13 \leq 1.2
$$

By completing the equation (20) with numbers

$\overline{\mathrm{Q}}_{0}=29,2 \mathrm{kN}, \Delta \mathrm{Q}_{\mathrm{trzecz}}=14,34 \mathrm{kN} \quad$ (spring group IV [3]), $\mathrm{Y}_{\mathrm{a}}=17.442 \mathrm{kN}$ results in:

$$
\left(\frac{17.442}{29.2-14.34}\right)=1.17 \leq 1.2
$$

By completing the equation (20) with numbers

$\overline{\mathrm{Q}}_{0}=29,2 \mathrm{kN}, \Delta \mathrm{Q}_{\mathrm{trzecz}}=14,47 \mathrm{kN} \quad$ (spring group III [3]), $\mathrm{Y}_{\mathrm{a}}=17.442 \mathrm{kN}$ results in:

$$
\left(\frac{17.442}{29.2-14.47}\right)=1.18 \leq 1.2
$$

By completing the equation (20) with numbers

$\overline{\mathrm{Q}}_{0}=29,2 \mathrm{kN}, \Delta \mathrm{Q}_{\mathrm{trzecz}}=14,65 \mathrm{kN}$ (spring group II [3]), $\mathrm{Y}_{\mathrm{a}}=17.442 \mathrm{kN}$ results in:

$$
\left(\frac{17.442}{29.2-14.65}\right)=1.19 \leq 1.2
$$

By completing the equation (20) with numbers $\overline{\mathrm{Q}}_{0}=29,2 \mathrm{kN}, \Delta \mathrm{Q}_{\mathrm{trzecz}}=14,79 \mathrm{kN}$ (spring group I [3]), $\mathrm{Y}_{\mathrm{a}}=17.442 \mathrm{kN}$ results in:

$$
\left(\frac{17.442}{29.2-14.79}\right)=1.21 \approx 1.2
$$

The permissible wheel load reduction $\Delta \mathrm{Q}_{\text {tdop }}$ for a rail tanker 406R can be determined using the modified equation (20) after substituting in values for $\mathrm{Y}=\mathrm{Y}_{\mathrm{a}}=17.442 \mathrm{kN}$ and: $\overline{\mathrm{Q}}_{0}=29,2 \mathrm{kN}$

$$
\Delta \mathrm{Q}_{\mathrm{DDOP}}=\bar{Q}_{0}-\frac{\mathrm{Y}_{\mathrm{a}}}{1.2}=29.2-\frac{17.442}{1.2}=14.66 \mathrm{kN}
$$

$$
\frac{Y_{a}}{Q_{r}}=\frac{Y_{a}}{\bar{Q}_{o}-\Delta Q_{t}}=\frac{17,442}{29,2-13,89}=1,13
$$

Wskaźnik wykolejenia jest mniejszy od przejazdu $\mathrm{w}$ warunkach quasi-statycznych tzn.1,2.

Względny wskaźnik odciążenia $\Delta \mathrm{q}$ wynosi:

$$
\Delta \mathrm{q}=\frac{\Delta \mathrm{Q}_{\mathrm{rz}}}{\overline{\mathrm{Q}}_{0}}=\frac{29,2-15,31}{29,2}=0,47
$$

Graniczny względny wskaźnik odciążenia koła $\lim \Delta \mathrm{q}$ wynosi:

$\lim \Delta \mathrm{q}=\frac{\lim \left(\frac{\mathrm{Y}}{\mathrm{Q}}\right)_{\mathrm{a}}-\frac{\mathrm{Y}_{\mathrm{a}}}{\overline{\mathrm{Q}}_{0}}}{\lim \left(\frac{\mathrm{Y}}{\mathrm{Q}}\right)_{\mathrm{a}}}=\frac{1,2-\frac{17,442}{29,2}}{1,2}=\frac{1,2-0,59}{1,2}=0,50$

Warunek $\Delta \mathrm{q}<\lim \Delta \mathrm{q}$ jest spełniony.

Podstawiając do wzoru (20) dane liczbowe

$\overline{\mathrm{Q}}_{0}=29,2 \mathrm{kN}, \Delta \mathrm{Q}_{\mathrm{trzecz}}=13,89 \mathrm{kN} \quad$ (podatność nominalna resorów), $\mathrm{Y}_{\mathrm{a}}=17,442 \mathrm{kN}$ otrzymuje się:

$$
\left(\frac{17,442}{29,2-13,89}\right)=1,13 \leq 1,2
$$

Podstawiając do wzoru (20) dane liczbowe

$\overline{\mathrm{Q}}_{0}=29,2 \mathrm{kN}, \Delta \mathrm{Q}_{\mathrm{trzecz}}=14,34 \mathrm{kN}$ (IV grupa resorów wg [3]), $Y_{a}=17,442 \mathrm{kN}$ otrzymuje się:

$$
\left(\frac{17,442}{29,2-14,34}\right)=1,17 \leq 1,2
$$

Podstawiaiac do wzoru (20) dane liczbowe

$\overline{\mathrm{Q}}_{0}=29,2 \mathrm{kN}, \Delta \mathrm{Q}_{\mathrm{trzecz}}=14,47 \mathrm{kN} \quad$ (III grupa resorów wg [3]), $Y_{\mathrm{a}}=17,442 \mathrm{kN}$ otrzymuje się:

$$
\left(\frac{17,442}{29,2-14,47}\right)=1,18 \leq 1,2
$$

Podstawiając do wzoru (20) dane liczbowe

$\overline{\mathrm{Q}}_{0}=29,2 \mathrm{kN}, \Delta \mathrm{Q}_{\mathrm{trzecz}}=14,65 \mathrm{kN} \quad$ (II grupa resorów wg [3]), $\mathrm{Y}_{\mathrm{a}}=17,442 \mathrm{kN}$ otrzymuje się:

$$
\left(\frac{17,442}{29,2-14,65}\right)=1,19 \leq 1,2
$$

Podstawiając do wzoru (20) dane liczbowe $\overline{\mathrm{Q}}_{0}=29,2 \mathrm{kN}, \Delta \mathrm{Q}_{\mathrm{trzecz}}=14,79 \mathrm{kN} \quad$ (I grupa resorów $\mathrm{wg}[3]), \mathrm{Y}_{\mathrm{a}}=17,442 \mathrm{kN}$ otrzymuje się:

$$
\left(\frac{17,442}{29,2-14,79}\right)=1,21 \approx 1,2
$$

Dopuszczalną wartość odciążenia koła $\Delta \mathrm{Q}_{\text {tdop }}$ dla wagonu cysterny 406R można wyznaczyć z przekształconego wzoru (20) po wstawieniu $\mathrm{Y}=\mathrm{Y}_{\mathrm{a}}=17,442 \mathrm{kN}$ oraz $\overline{\mathrm{Q}}_{0}=29,2 \mathrm{kN}$

$$
\Delta \mathrm{Q}_{\mathrm{tDOP}}=\bar{Q}_{0}-\frac{\mathrm{Y}_{\mathrm{a}}}{1,2}=29,2-\frac{17,442}{1,2}=14,66 \mathrm{kN}
$$




\section{Summary of the conducted analyses 3.1. General remarks}

According to the analysis, the 406R wagon with $2 \mathrm{a}^{*}=6,8 \mathrm{~m}$ base on 1XTa bogeys is safe when driving on a warped track. Value of the derailment coefficient applicable for quasi-static conditions is not exceeded. In the event of any structural modification by means of clearance adjustment in a positive tolerance, it may be considered that the loss of derailment safety due to increased spring rigidity can be compensated by additional clearance adjustment in the upper tolerance range. This construction would increase the safety reserve and increase the probability of "safe driving of the rail tanker" through warped tracks. The derailment coefficient of 1.2 adapted by ORE/ERRI B55 Rp. 8 [12], guarantees a safe ride with internationally accepted probability of $95 \%$ (ORE/ERRI, UIC).

Increased torsion stiffness values of the 1XTa bogey are found in other rail tankers tested and presented in the ORE/ERRI B55Rp.8 [12]. An example of this may be the 422R (Uahs) rail tanker, with the following parameters: $\quad 2 \mathrm{a}^{*}=6.8 \quad \mathrm{~m}, \quad \mathrm{c}_{\mathrm{tA}(2 \mathrm{a}}{ }^{*}=4.02 \quad \mathrm{kN} / \%$, $\mathrm{c}_{\mathrm{tA}\left(2 \mathrm{a}^{*}\right)}=1.20 \mathrm{kN} / \%$. This wagon with a significantly higher torsional stiffness on the base of the bogey has a lower vertical wheel load, which increases its sensitivity to derailment (the base load is $19 \%$ lower than for the 406R wagon). Wagons with lower mass on the same bogeys are more susceptible to derailment. The above derailment safety analysis for driving on warped track was carried out for rail tank wagon type 406R. Similar conclusions apply to wagons used by carriers i.e.:

$>$ for wagons $406 \mathrm{Rb}$ with a base of $2 \mathrm{a}^{*}=6.8 \mathrm{~m}$, for the transport of gasoline, diesel fuel, light and heavy fuel oils and others,

$>$ for wagons $406 \mathrm{RU}$ with a base of $2 \mathrm{a}^{*}=6.8 \mathrm{~m}$, for the transport of liquefied gases,

$>$ for wagons $452 \mathrm{R}$ with a base of $2 \mathrm{a}^{*}=9.7 \mathrm{~m}$ for the transport of light petroleum products.

\subsection{The effect of the spring height on its properties and function in the running gear}

The data given in Table 1 shows, that springs of different height, divided in ranges of $196 \div 198 \mathrm{~mm}$, $198 \div 200 \mathrm{~mm}, 200 \div 202 \mathrm{~mm}, 202 \div 204 \mathrm{~mm}$ are present for different flexibility groups I to IV. Therefore, it is not possible to draw a conclusion on the linear character of the spring flexibility to its height. The phenomena observed in operation are known to be opposite to one of the observed, namely the loss of height, and it results uniquely from the system of forces acting on the spring. "Spring collapse", i.e. loss of height caused by permanent deformation of the leaf springs, is a more serious phenomenon, as it can cause a gradual decrease in the clearance between the spring band and the shock absorber, and given the significant dynamic surpluses for freight wagons reaching $1.5 \div 1.6$

\section{Podsumowanie z przeprowadzonych analiz 3.1. Uwagi ogólne}

Z przedstawionej analizy wynika, że wagon 406R o bazie $2 \mathrm{a}^{*}=6,8 \mathrm{~m}$ na wózkach $1 \mathrm{XTa}$ jest bezpieczny podczas jazdy na wichrowatym torze. Współczynnik (lub wskaźnik) wykolejenia obowiązujący dla warunków quasi-statycznych nie jest przekroczony. W przypadku ewentualnego zabiegu konstrukcyjnego polegającemu na regulacji luzów w dodatniej tolerancji można uważać, że ubytek zapasu bezpieczeństwa przed wykolejeniem spowodowany zwiększoną sztywnością może być rekompensowany dodatkowo regulacją luzów w górnym zakresie tolerancji. Ten zabieg konstrukcyjny pozwoliłby zwiększyć zapas bezpieczeństwa oraz zwiększenie prawdopodobieństwa „bezpiecznej jazdy wagonu cysterny" przez tory wichrowate. Wskaźnik wykolejenia przyjęty na poziomie 1,2 gwarantuje zgodnie $\mathrm{z}$ raportem ORE/ERRI B55 Rp.8 [12], bezpieczną jazdę $\mathrm{z}$ uznanym międzynarodowo prawdopodobieństwem 95\% (ORE/ERRI, UIC).

Zwiększone wartości sztywności skrętne, wózka 1XTa są spotykane w innych przebadanych wagonach cysternach i przedstawione $w$ raporcie ORE/ERRI B55Rp.8 [12]. Przykładem tego może być wagon cysterna 422R (Uahs), która posiada następujące parametry: $\quad 2 \mathrm{a}^{*}=6,8 \quad \mathrm{~m}, \quad, \quad \mathrm{c}_{\mathrm{tA}(2 \mathrm{a})}{ }^{*}=4,02 \quad \mathrm{kN} / \%$, $\mathrm{c}_{\mathrm{tA}\left(2 \mathrm{a}^{*}\right)}=1,20 \mathrm{kN} / \%$. Wagon ten przy znacznie większej sztywności skrętnej na bazie wózka posiada mniejszy nacisk pionowy koła, co zwiększa jego wrażliwość na wykolejania (nacisk bazowy jest mniejszy od wagonu 406R o 19\%). Wagony o mniejszej masie własnej na tych samych wózkach są bardziej wrażliwe na wykolejenia. Powyższa analiza bezpieczeństwa przed wykolejeniem podczas przejazdu przez tory wichrowate została przeprowadzona dla wagonów cystern typu 406R. Podobne wnioski obowiązuja dla wagonów cystern użytkowanych przez przewoźników tzn.:

$>$ dla wagonów typu $406 \mathrm{Rb}$ o bazie $2 \mathrm{a}^{*}=6,8 \mathrm{~m}$, do przewozu benzyny, oleju napędowego, oleju opałowego lekkiego i ciężkiego i innych,

$>$ dla wagonów typu 406RU o bazie $2 \mathrm{a}^{*}=6,8 \mathrm{~m}$, do przewozu gazów skroplonych,

$>$ dla wagonów typu $452 \mathrm{R}$ o bazie $2 \mathrm{a}^{*}=9,7 \mathrm{~m}$ do przewozu lekkich produktów naftowych.

\subsection{Wpływ wysokości resoru na jego własności oraz pelnioną funkcję w ukladzie biegowym}

Jak wynika $\mathrm{z}$ danych zamieszczonych $\mathrm{w}$ tablicy 1 różne wysokości resorów podzielone na przedziały o wartościach $196 \div 198 \mathrm{~mm}, 198 \div 200,200 \div 202 \mathrm{~mm}$, $202 \div 204 \mathrm{~mm}$ występują dla różnych grup podatności od I do IV. Nie można więc wyciagnąc wniosku o liniowym charakterze zależności podatności od wysokości resoru. Ze zjawisk obserwowanych w 
[13] it may lead to local damage to the bogey side sill. Thus, the spring can be fitted in a 1XTa bogey (as long as other geometric dimensions are within the tolerances provided), provided the pickup dimensions are correct (the height of the center pivot point and the height between the spring clamp and the shock absorbers).

\subsection{Suspension spring operational criteria}

The analysis shows that due to unfulfilled spring criteria i.e. deflection $f_{m}$ under test force $F_{m}$ there is a spread of flexibility which exceeds the admissible values in the four groups of springs. The permissible spread of flexibility values is $\pm 8 \%$ in accordance with PN-61/K-88181 [13], card UIC 821 [8] and additional regulations WT-2 cz.4a-4c, OW-305 [14]. This conclusion was drawn on the basis of a load curve rather than a hysteresis loop, which is the result of friction between the leaves. The friction force during operation can fluctuate within fairly large limits depending on the coefficient of friction between the spring leaves. As defined in [1] the relation between the loading force, e.g. $F_{m}$, and the friction force $T$ can be determined using the formula:

$$
\varphi_{\mathrm{t}}=\frac{\mathrm{T}}{\mathrm{F}_{\mathrm{m}}}
$$

where:

$$
\varphi_{t}-\text { coefficient of relative friction. }
$$

The coefficient of relative friction can be determined from:

$$
\varphi_{\mathrm{t}}=2 \mu \cdot(\mathrm{n}-1) \cdot \frac{\mathrm{h}}{\mathrm{L}}
$$

where:

$\mu$ - coefficient of friction between springs, if there is no experimental data, then it is assumed to be:

$>$ in the range $\mu=0.3 \div 0.4$ for graphite coated leaves

$>\mu=0.8$ for non-coated leaves,

$\mathrm{n}$ - number of leaves in the spring,

$\mathrm{h}$ - leaf spring thickness,

$\mathrm{L}$ - leaf spring length.

The coefficient of relative friction for $\mu=0.4$ (coated leaves), $\mathrm{n}=8, \mathrm{~L}=1120 \mathrm{~mm}$ and $\mathrm{h}=16 \mathrm{~mm}$ after substituting into (41) equals:

$$
\varphi_{t}=2 \cdot 0.4 \cdot(8-1) \cdot \frac{16}{1120}=0.08
$$

The coefficient of relative friction for $\mu=0.8$ (noncoated leaves), $\mathrm{n}=8, \mathrm{~L}=1120 \mathrm{~mm}$ and $\mathrm{h}=16 \mathrm{~mm}$ after substituting into (41) equals:

$$
\varphi_{\mathrm{t}}=2 \cdot 0.8 \cdot(8-1) \cdot \frac{16}{1120}=0.16
$$

The friction force between the leaves in the non-coated leaf spring at the test load $\mathrm{F}_{\mathrm{m}}=134 \mathrm{kN}$ in accordance with the formula (40) equals $10.72 \mathrm{kN}$, while for the coated leaf spring $21.44 \mathrm{kN}$. The shown hysteresis eksploatacji znane jest raczej zjawisko przeciwne do stwierdzonego, a mianowicie utrata wysokości, czyli „siadanie resoru” i wynika ono jednoznacznie z układu sił działających na resor. „Siadanie resoru”, czyli utrata wysokości spowodowana odkształceniem trwałym piór resoru jest zjawiskiem znacznie groźniejszym, gdyż może spowodować stopniowe zmniejszenie luzu pomiędzy opaską resoru, a odbijakiem i przy znacznych nadwyżkach dynamicznych przewidzianych dla wagonów towarowych kształtujących się na poziomie 1,5 $\div 1,6$ [13] może dojść do lokalnego uszkodzenia pasa ostojnicy wózka. W związku z powyższym należy wnioskować, że o ile resor taki można zamontować w wózku 1XTa (pozostałe wymiary geometryczne mieszczą się $\mathrm{w}$ przewidzianych tolerancjach), pod warunkiem że wymiary odbiorcze wózka są prawidłowe (wysokość gniazda skrętu oraz wysokości pomiędzy opaskami resorowymi oraz odbijakami).

\subsection{Kryterium odbiorcze resorów}

$\mathrm{Z}$ przeprowadzonej analizy wynika, że $\mathrm{z}$ racji niespełnionego kryterium odbiorczego tzn. ugięcia $f_{m}$ pod siła próbna $F_{m}$ w czterech grupach resorów występuje rozrzut podatności, który przekracza wartości dopuszczalne. Dopuszczalny rozrzut podatności wynosi \pm \% zgodnie z PN-61/K-88181 [13], kartą UIC 821 [8] oraz przepisami naprawczymi WT-2 cz.4a-4c, OW-305 [14] Wniosek ten został wyciagnnięty w oparciu o krzywą dociążeniowa, a nie występującą pętlę histerezy, która jest wynikiem tarcia pomiędzy piórami. Siła tarcia podczas eksploatacji może ulegać wahaniom w dość znacznych granicach zależnych między innymi od współczynnika tarcia pomiędzy piórami resoru. Na podstawie [1] zależność pomiędzy siłą obciążająca $n p . F_{m}$, a siłą tarcia $T$ można określić wg wzoru:

$$
\varphi_{\mathrm{t}}=\frac{\mathrm{T}}{\mathrm{F}_{\mathrm{m}}}
$$

gdzie:

$\varphi_{\mathrm{t}}-$ współczynnik tarcia względnego.

Współczynnik tarcia względnego można wyznaczyć z zależności:

$$
\varphi_{\mathrm{t}}=2 \mu \cdot(\mathrm{n}-1) \cdot \frac{\mathrm{h}}{\mathrm{L}}
$$

gdzie:

$\mu$ - współczynnik tarcia pomiędzy piórami resoru, jeżeli brak jest danych doświadczalnych, to przyjmuje się go:

$>\mathrm{w}$ granicach $\mu=0,3 \div 0,4$ dla piór smarowanych grafitem oraz

$>\mu=0,8$ dla piór nie smarowanych,

$\mathrm{n}$ - ilość piór resorowych,

$\mathrm{h}$ - grubość pióra resorowego,

L - długość pióra resorowego.

Współczynnik tarcia względnego dla $\mu=0,4$ (pióra smarowane), $\mathrm{n}=8, \mathrm{~L}=1120 \mathrm{~mm}$ oraz $\mathrm{h}=16 \mathrm{~mm}$ po podstawieniu do wzoru (41) wynosi: 
loop indicates that the same deflection can be obtained when the spring is released at $123.28 \mathrm{kN}$ (coated springs) or at $112.54 \mathrm{kN}$. If such severe scattering can be caused by the friction force alone, then the result of the flexibility spread is no longer surprising. If the four springs groups flexibilities were to be correctly represented in tests in accordance with the UIC 821 [8] card, it should be considered that the calculated flexibility values at force $F_{m}$ are correct. Due to the increase in height between the clamps and the shock absorbers from $74_{-6}^{+2} \mathrm{~mm}$ to $80^{+6} \mathrm{~mm}$ the force $\mathrm{F}_{\mathrm{m}}$ must be increased from $134 \mathrm{kN}$ to $142 \mathrm{kN}$, and the deflection adjusted to $92 \mathrm{~mm} \pm 8 \%$.

\section{CONCLUSIONS}

1. In order to test the flexibility as specified in UIC 821 [8], the deflection under test load $\mathrm{F}_{\mathrm{m}}$, frictional force $\mathrm{T}$ possible testing of 20 selected springs (from each of the flexibility groups listed in this study as I to IV) could be considered in any laboratory with proper accreditation. The tests could be carried out in parallel with the safety tests before derailing the rail tanker.

2. As a result of the analysis it is possible to allow the use of springs groups III and IV in the described rail tankers. This decision should be supported with:

$>$ characteristics tests of 20 leaf springs, if performer, as well as

$>$ rail tanker $406 \mathrm{Rb}$ derailment safety study performed in accordance with PN-EN 14363:2016 [9].

3. The next structural adjustment to be performed for the rail tankers with more rigid springs is to increase the clearance of the side slips according to the following rule.

The clearance on the side slips should be as much as the base of the wagon in $\mathrm{mm}$ so:

$>$ for a $406 \mathrm{Rb}$ and $406 \mathrm{Ru}$ wagons with a base of $2 \mathrm{a}^{*}=6.8 \mathrm{~m}$ the clearance should be $7^{+2} \mathrm{~mm}$, thus it is proposed to use a clearance of $9 \mathrm{~mm}$ on all wagons of this type,

$>$ for wagons $452 \mathrm{R}$ with a base of $2 \mathrm{a}^{*}=9.7 \mathrm{~m}$ the clearance should be $10^{+2} \mathrm{~mm}$, thus it is proposed to use a clearance of $12 \mathrm{~mm}$ in all wagons of this type.

4. Increased clearance in the side slips increases the value of $E_{i}$ (between wagon center pivots) and $E_{a}$ (outside the center pivots) from the reference profile of the kinematic gauge in accordance with UIC 505-1 [5] and reduces the permissible gauge of the wagon. However, as the analytical work shows, the decrease in clearance should not jeopardize the safe

$$
\varphi_{t}=2 \cdot 0,4 \cdot(8-1) \cdot \frac{16}{1120}=0,08
$$

Współczynnik tarcia względnego dla $\mu=0,8$ (pióra nie smarowane), $\mathrm{n}=8, \mathrm{~L}=1120 \mathrm{~mm}$ oraz $\mathrm{h}=16 \mathrm{~mm}$ po podstawieniu do wzoru (41) wynosi:

$$
\varphi_{\mathrm{t}}=2 \cdot 0,8 \cdot(8-1) \cdot \frac{16}{1120}=0,16
$$

Siła tarcia pomiędzy piórami $\mathrm{w}$ resorze nie smarowanym przy obciążeniu próbnym $\mathrm{F}_{\mathrm{m}}=134 \mathrm{kN}$ wynosi zgodnie ze wzorem (40) $10,72 \mathrm{kN}$, natomiast dla resoru nie smarowanego 21,44 $\mathrm{kN}$. Z przedstawionej pętli histerezy wynika, że takie samo ugięcie może być osiagnięte podczas odciążenia resoru przy sile $123,28 \mathrm{kN}$ (resory smarowane) lub przy sile $112,54 \mathrm{kN}$. Jeśli tak poważne rozrzuty ugięcia może powodować sama siła tarcia, to wynik rozrzutu podatności nie jest żadnym zaskoczeniem. Jeśli cztery grupy podatności resorów powtórzyłyby się przy poprawnie wykonanych próbach zgodnych $\mathrm{np}$. z karta UIC 821 [8] to należy uznać, że wyliczone rozrzuty podatności przy sile $\mathrm{F}_{\mathrm{m}}$ są prawidłowe. $\mathrm{W}$ związku ze zwiększeniem wysokości pomiędzy opaską resorową a odbijakiem z $74_{-6}^{+2} \mathrm{~mm}$ do $80^{+6} \mathrm{~mm}$ należy zwiększyć siłę $\mathrm{F}_{\mathrm{m}}$ ze $134 \mathrm{kN}$ do $142 \mathrm{kN}$, natomiast ugięcie skorygować na $92 \mathrm{~mm} \pm 8 \%$.

\section{WNIOSKI}

1. W celu sprawdzenia podatności zgodnie z kartą UIC 821 [8], ugięcia pod obciążeniem próbnym $\mathrm{F}_{\mathrm{m}}$, siły tarcia $\mathrm{T}$ można rozważyć ewentualne podjęcie badań 20-tu wytypowanych resorów (z każdej grupy podatności wymienionej $\mathrm{w}$ tym opracowaniu jako I do IV) w laboratorium badawczym, posiadającym akredytację. Badania mogłyby być przeprowadzone równolegle $\mathrm{z}$ badaniami bezpieczeństwa przed wykolejeniem wagonu cysterny.

2. W wyniku przeprowadzonej analizy istnieje możliwość dopuszczenia grup resorów III i IV do ww. wagonów cystern.Decyzję tę należy poprzeć:
$>$ ewentualnie podjętymi badaniami doświadczalnymi charakterystyk 20-tu resorów oraz
$>$ badaniami bezpieczeństwa przed wykolejeniem dla wagonu cysterny $406 \mathrm{Rb}$ przeprowadzonymi zgodnie z PN-EN 14363:2016 [9].

3. Kolejnym zabiegiem konstrukcyjnym jaki należy wykonać przy wagonach cysternach ze sztywniejszymi resorami jest zwiększenie luzów na ślizgów bocznych zgodnie $\mathrm{z}$ następującą regułą. 
operation of the wagon (no collision of the socalled "critical points" of the wagon with the fixed devices located beyond the vehicle gauge). The principle to establish the clearance level in the upper tolerance of a set value is justified by the fact that the wear processes that takes place on the working surfaces of the center pivot-center pivot point contributes to reducing the clearance on the side slips.

5. The modernization of the 1 XTa bogey would be a significant upgrade, which would include, among others, the use of parabolic suspension springs with progressive characteristics. Then the spring has soft characteristics for the empty wagon and a hard characteristics for the loaded wagon. This would undoubtedly increase the safety of freight cars.

\section{BIBLIOGRAPHY BIBLIOGRAFIA}

[1] Gross. S.: Berechnung und Gestaltung von Metallfedern. Konstruktionsbücher. Springer-Verlag Berlin. Göttingen. Heidelberg.1960. Dritte Auflage.

[2] Sobaś M: Zawieszenia i układy biegowe pojazdów szynowych. Wydawnictwo IPS Tabor. 2014.

[3] Sobaś M.: Bezpieczeństwo jazdy wagonów towarowych wyposażonych $w$ wózki 1 XTa z resorami piórowymi. Pojazdy Szynowe nr 2/2017

[4] Sobaś M: bezpieczeństwo jazdy wagonów towarowych wyposażonych $w$ wózki 1 XTa z resorami piórowymi w aspekcie wytrzymałości i przejazdu przez tory wichrowate. Pojazdy Szynowe nr 3/2017.

[5] Karta UIC 505-1: Pojazdy kolejowe. Skrajnie pojazdów. 10-te wydanie z maja 2006.

[6] Karta UIC 517: Wagony towarowe. Części zawieszenia resorowego. Normalizacja. 6-te wydanie $z$ 1.01.1979, nowy nakład z 1.01.1989 z 10-ta zmiana $z$ 1.07.1997

[7] Karta UIC 820: Warunki techniczne dla dostawy stali sprężynowej płaskiej dla resorów piórowych i sprężyn stożkowych. 6-te wydanie z grudnia 2003.

[8] Karta UIC 821: Warunki techniczne dostawy resorów piórowych dla pojazdów. 6-te wydanie $z$ listopada 2003.

[9] PN-EN 14363:2016: Kolejnictwo. Badania własności dynamicznych przed dopuszczeniem pojazdów szynowych. Badania właściwości biegowych $i$ próby stacjonarne.

[10] Raport ORE/ERRI B12/Rp14/D: Vereinheitlichung der Güterwagen. Standard-Drehge-stell. Bericht 14. Listopad 1967.

[11] Raport ORE/ERRI B12.Bericht Nr. 25: Spezifische Federung der Trapezfedern. Utrecht. Wrzesień 1986 (2Ausgabe).

[12] Raport ORE/ERRI B55 Rp.8: Sicherheit gegen Entgleisen von Güterwagen in Gleisverwindungen. Utrecht. April 1983.

[13] PN-61/K-88181: Tabor kolejowy. Wagony towarowe. Resory piórowe.

[14] WT-2 cz.4a-4c, OW305: Warunki techniczne kwalifikacji do naprawy, naprawy i odbioru po naprawie sprężyn nośnych wagonów towarowych.
Luz na ślizgach bocznych powinien wynosić tyle ile baza wagonu w mm i tak:

$>$ dla wagonu 406Rb oraz wagonu 406Ru o bazie $2 \mathrm{a}^{*}=6,8 \mathrm{~m}$ luz powinien wynosić $7^{+2}$ $\mathrm{mm}, \mathrm{w}$ związku z powyższym proponuje się na wszystkich wagonach tego typu zastosowanie luzów wynoszących $9 \mathrm{~mm}$,

$>$ dla wagonu $452 \mathrm{R}$ o bazie $2 \mathrm{a}^{*}=9,7 \mathrm{~m}$ luz powinien wynosić $10^{+2} \mathrm{~mm}$, w związku z powyższym proponuje się na wszystkich wagonach tego typu zastosowanie luzów wynoszących $12 \mathrm{~mm}$.

4. Zwiększone luzy na ślizgach bocznych powodują zwiększenie wartość zwężeń $E_{i}$ (między czopami skrętu wagonu) i $\mathrm{E}_{\mathrm{a}}$ (na zewnątrz czopów skrętu wagonu) od zarysu odniesienia skrajni kinematycznej zgodnie $\mathrm{z}$ kartą UIC 505-1 [5] i zmniejszenie dopuszczalnego zarysu wagonu. Jak wynika jednak z przeprowadzonych prac analitycznych, wzrost zwężeń $\mathrm{z}$ tego powodu nie powinien zagrażać bezpiecznej eksploatacji wagonu (brak kolizji tzw. „punkty krytyczne zarysu” wagonu nie wchodzą $\mathrm{w}$ kolizję $\mathrm{z}$ urządzeniami stałymi, umieszczonymi, poza zarysem skrajni budowli). Zasada, aby ustalać poziom luzów w górnej tolerancji ustalonej wartości jest uzasadniona tym, że procesy zużycia, które odbywają się na powierzchniach współpracy pomiędzy powierzchnią czop skrętu-gniazdo skrętu, przyczyniają się do zmniejszenia luzów na ślizgach bocznych.

5. Istotnym postępem modernizacyjnym byłoby modernizacja wózka 1XTa, która polegałaby między innymi na zastosowaniu resorów parabolicznych z progresywną charakterystyką. Wówczas resor ma miękką charakterystykę $\mathrm{w}$ stanie próżnym wagonu oraz twardą charakterystykę $\mathrm{w}$ stanie ładownym wagonu. Zwiększyłoby to niewątpliwie bezpieczeństwo jazdy wagonów towarowych. 